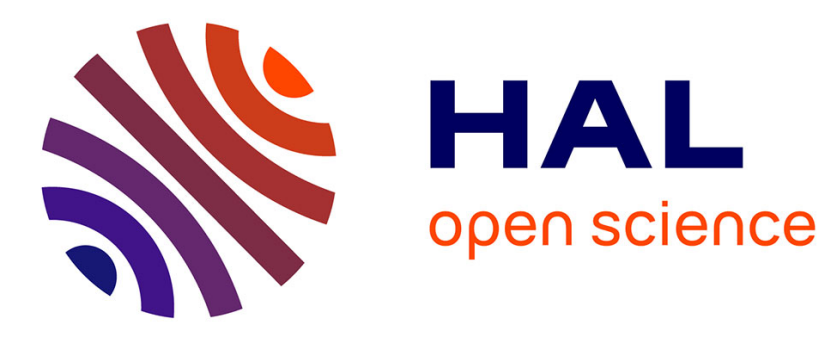

\title{
EGFR inhibition by curcumin in cancer cells: a dual mode of action.
}

Marcelina Starok, Pascal Preira, Muriel Vayssade, Karsten Haupt, Laurence Salome, Claire Rossi

\section{- To cite this version:}

Marcelina Starok, Pascal Preira, Muriel Vayssade, Karsten Haupt, Laurence Salome, et al.. EGFR inhibition by curcumin in cancer cells: a dual mode of action.. Biomacromolecules, 2015, 16 (5), pp.1634-1642. 10.1021/acs.biomac.5b00229 . hal-03003753

\section{HAL Id: hal-03003753 \\ https://hal.science/hal-03003753}

Submitted on 13 Nov 2020

HAL is a multi-disciplinary open access archive for the deposit and dissemination of scientific research documents, whether they are published or not. The documents may come from teaching and research institutions in France or abroad, or from public or private research centers.
L'archive ouverte pluridisciplinaire HAL, est destinée au dépôt et à la diffusion de documents scientifiques de niveau recherche, publiés ou non, émanant des établissements d'enseignement et de recherche français ou étrangers, des laboratoires publics ou privés. 
3 Marcelina Starok ${ }^{1}$, Pascal Preira ${ }^{2,3}$, Muriel Vayssade ${ }^{4}$, Karsten Haupt $^{1}$, Laurence Salomé2,3, Claire Rossi ${ }^{1} *$

$6 \quad{ }^{1}$ Sorbonne universités, Université de Technologie de Compiègne, CNRS, Laboratoire de Génie

7 enzymatique et cellulaire, Rue Roger Couttolenc, CS 60319, F-60203 Compiègne Cedex, France.

$8{ }^{2}$ CNRS ; Institut de Pharmacologie et de Biologie Structurale, 205 route de Narbonne, F-31077

9 Toulouse, France

$10 \quad{ }^{3}$ Université de Toulouse, UPS, IPBS, F-31077 Toulouse, France

$11{ }^{4}$ Sorbonne universités, Université de Technologie de Compiègne, CNRS, Laboratoire de

12 Biomécanique et Bioingénierie, Rue Roger Couttolenc, CS 60319, F-60203 Compiègne Cedex,

13 France 


\section{ABSTRACT}

2 Epidermal Growth Factor Receptor (EGFR) is an important target of anticancer therapy.

3 Nowadays the search for new molecules inhibiting this receptor is turning towards natural

4 substances. One of the most promising natural compounds that have shown an anti-EGFR activity

5 is curcumin, a polyphenol found in turmeric. Its effect on the receptor kinase activity and on the

6 receptor autophosphorylation has been already described, but the mechanism of how curcumin

7 interacts with EGFR is not fully elucidated. We demonstrate that the mode of action of curcumin

8 is dual. This polyphenol is able to inhibit directly but partially the enzymatic activity of the EGFR

9 intracellular domain. The present work shows that curcumin also influences the cell membrane

10 environment of EGFR. Using biomimetic membrane models, we show that curcumin insertion into

11 the lipid bilayer leads to its rigidification. Single particle tracking analyses performed in the

12 membrane of A431 cancer cells confirmed that this effect of curcumin on the membrane slows

13 down the receptor diffusion. This is likely to affect the receptor dimerization and in turn its

14 activation.

15 KEYWORDS: Epidermal growth factor receptor, curcumin, autophosphorylation, tyrosine kinase

16 inhibition, human epidermoid carcinoma cells 


\section{INTRODUCTION}

2

3 The epidermal growth factor receptor (EGFR), also known as ErbB1 or HER1, is one of the most

4 studied members of the receptor tyrosine kinase superfamily. ${ }^{1}$ EGFR is a central element for

5 cellular signal transduction and a regulator of essential epidermal functions, as proliferation,

6 survival, differentiation or migration. ${ }^{2-4}$ EGFR plays also a critical role in a variety of

7 pathophysiological phenomena, particularly in cancer development. ${ }^{5-7}$

8 The EGF receptor is characterized by a modular structure consisting of an extracellular ligand-

9 binding domain, a single hydrophobic transmembrane region, and an intracellular part including

10 the tyrosine kinase domain. In the generally accepted model of EGFR activation, the

11 oligomerization of protein monomers is considered as a key intermediate step between binding of

12 the growth factor and activation of the tyrosine kinase. Ligand binding to the extracellular domain

13 stabilizes the receptor in its extended conformation, which promotes its dimerization. ${ }^{8-10}$ In this

14 conformation, the proximity of the tyrosine kinase domains promotes the transphosphorylation of

15 tyrosine residues on both monomers, which creates docking sites for enzyme substrates -

16 intracellular proteins participating in signal transduction. ${ }^{11}$ Numerous reports based on different

17 approaches are supporting this concept, such as the observation of cluster formation by single

18 molecule tracking or FRET. ${ }^{12}$

19 The immense interest in this receptor was inspired by the finding that its excessive signalling,

20 related to overexpression, mutations or autocrine stimulation, disrupts equilibrium between cell

21 growth and apoptosis, leading to the development of a variety of solid tumors. ${ }^{5,7,13,14}$ The most

22 aggressive forms of tumors, correlated with poor prognosis and reduced chemosensitivity, are

23 usually associated with EGFR overexpression. ${ }^{7,15}$ For that reasons EGFR has been a key target of

24 the pharmaceutical industry since its first clinical trial performed in $1990 .{ }^{13,16,17}$ Two major 
1 approaches have been developed for targeting EGFR which demonstrated benefits in clinical trials:

2 monoclonal antibodies (mAbs), which interact with the extracellular domain of the protein making

3 ligand binding and consecutive dimerization impossible, and tyrosine kinase inhibitors (TKIs),

4 usually targeting the ATP binding site of the tyrosine kinase domain. Although a number of mAbs

5 or TKIs are commercially available for the treatment of a variety of cancers, ongoing efforts are

6 made by academic research and industrial pharmaceutical $R \& D$ to develop new drugs and/or new

7 strategies in order to reduce side-effects or resistance development. ${ }^{18,19}$

8 Natural substances are the source of promising molecules with anticancer potential, such as

9 epigallocatechin gallate, delphinidin or butein. ${ }^{20,}{ }^{21}$ Among the polyphenols that inhibit EGFR

10 signaling, the most widely studied is curcumin, found in the rhizomes of Curcuma longa

11 (turmeric). ${ }^{22-26}$ Curcumin exhibits remarkable pharmacological activities, such as anti-

12 inflammatory, anti-neoplastic, anti-oxidant and chemopreventive effects, and has been shown to

13 be pharmacologically safe even at high doses (12 g/day). ${ }^{27-29}$ Curcumin action includes the

14 regulation of signal transduction pathways and the inhibition of enzymes and key transcription

15 factors. ${ }^{30}$ After treatment with curcumin, cancer cells display several features typical of apoptotic

16 cells. ${ }^{31,32}$ Inhibitory effect of curcumin on ligand-induced activity of EGFR was proven by studies

17 on living cells, on proteins reconstituted in liposomes and on isolated proteins. ${ }^{23-25}$ However, the

18 exact mode of action of this molecule is not fully understood. ${ }^{33}$ The present work is scrutinizing

19 the mechanism of early curcumin action on EGFR autophosphorylation by different approaches:

20 the effect of curcumin on EGFR activation was studied in vitro on separate EGFR domains and on

21 human epidermoid carcinoma cells. The impact of curcumin on the fluidity of the lipid bilayer,

22 which constitutes the direct environment of EGFR, was investigated as well. The results indicate 
1 a dual mode of action of curcumin, and thus contribute to our understanding of the interaction of 2 curcumin with the cell membrane, and of receptor activity modulation. 


\section{MATERIALS AND METHODS}

Cell culture. Human epidermoid carcinoma cell line A-431 was purchased from American Type Culture Collection (ATCC ${ }^{\circledR}$ CRL-1555) and cultured in high glucose (4500 mg/L) Dulbecco's Modified Eagle's Medium (DMEM) (D6429 Sigma, France) supplemented with $10 \%$ (v/v) fetal bovine serum (FBS) (Gibco, France) and $1 \%$ (v/v) penicillin/ streptomycin (Gibco, France). Routinely cells were kept in a humidified incubator at $37^{\circ} \mathrm{C}$ with $5 \% \mathrm{CO}_{2}$. The culture medium was changed three times a week. Subculturing was performed once a week, using a standard trypsinization protocol with $0.25 \%$ trypsin-EDTA (1X) (Gibco). For standard culture, cells were seeded into $75 \mathrm{~cm}^{2}$ flasks (Falcon) at 1500000 cells per flask. For the ELISA based assay cells were cultured in 96-well black cell culture plates with clear bottom (BD Falcon) at 35000 cells/well unless other indicated. Since curcumin stock solution was prepared in DMSO, control experiments were performed with DMSO $(<0.04 \%$ v/v), no DMSO effect on EGFR phosphorylation could be observed (data not shown).

Phospho-EGFR cell-based ELISA assay. Cell-based immunoassay allows for simultaneous quantification of the phosphorylated EGFR (Y1068) and total EGFR (pan-protein) which is used for normalization of the results. The method does not require cell lysis and the assay was performed directly in the culture plate after cell fixation and permeabilization. A431 cells were seeded at 35000 cells/well in the black cell culture 96-well plates with clear bottom (BD Falcon). After 24 $\mathrm{h}$ of growth, complete growth medium was replaced by the serum free medium for the following $24 \mathrm{~h}$, preceded by a single wash with PBS. Next, serum free medium containing different concentrations of curcumin was introduced into the culture for $4 \mathrm{~h}$ and then $100 \mathrm{ng} / \mathrm{ml}$ of EGF (Sigma-Aldrich) in serum free medium was added for an additional hour in the dark. Both incubation steps were performed at $37^{\circ} \mathrm{C}$ in $5 \% \mathrm{CO}_{2}$ atmosphere. Afterwards the cells were fixed 
1 with paraformaldehyde and immediately treated as described in the Human Phospho-EGFR/

2 ErbB1 (Y1068) cell-based ELISA protocol (R\&D Systems). The fluorescence intensity was read

3 at indicated wavelengths using a Cary Eclipse Fluorescence Spectrophotometer (Agilent

4 Technologies) equipped with a plate reader. The phosphorylation levels are expressed as

5 normalized relative fluorescence units (RFU).

6 Tyrosine kinase assay. EGFR tyrosine kinase domain (SRP5023, Sigma) was diluted from the

7 stock at $0.1 \mu \mathrm{g} / \mathrm{ml}$ to the concentration $140 \mathrm{pM}$ with Kinase Reacting Buffer provided in the

8 Universal Tyrosine Kinase Assay Kit (Takara, Ozyme). Curcumin was added at the desired

9 concentration to the solution and the reaction was initiated by addition of ATP. The

10 phosphorylation reaction was performed in the dark in order to prevent curcumin degradation and

11 was stopped after $30 \mathrm{~min}$. Within this time frame, less than $5 \%$ of curcumin decomposed (as judged

12 from UV/Vis spectra). Further steps were exactly as in the manual provided by manufacturer.

13 Absorbance was measured using a spectrophotometer equipped with a plate reader (Dynex MRX

14 Microplate Reader). The data were analyzed based on a calibration curve prepared with the

15 standard included in the set.

16 Biacore-based competition assay. Surface plasmon resonance (SPR) binding experiments were

17 performed on a BIAcore T100 instrument (GE Healthcare) at $25^{\circ} \mathrm{C}$ in $10 \mathrm{mM}$ HEPES buffer $\mathrm{pH}$

187.4 , containing $150 \mathrm{mM} \mathrm{NaCl}, 3.4 \mathrm{mM}$ EDTA and $0.005 \%$ Tween-20. Human recombinant EGF

19 (R\&D Systems) was coupled to a CM5 BIAcore sensor chip as follows: the chip was activated

20 with a freshly prepared 1:1 (v/v) mix of 0.4 M 1-Ethyl-3-(dimethylaminopropyl) carbodiimide

21 (EDC) with $0.1 \mathrm{M} N$-hydroxysuccinimide (NHS) for 7 min at $10 \mu \mathrm{l} / \mathrm{min}$. EGF (200 $\mu \mathrm{g} / \mathrm{ml}$ in 10

$22 \mathrm{mM}$ sodium acetate $\mathrm{pH} 4.0$ ) was then injected on the activated surface at $10 \mu \mathrm{l} / \mathrm{min}$ for $10 \mathrm{~min}$. An

23 EGF-free control surface was prepared with the same immobilization procedure, but injecting 
1 buffer instead of EGF. Recombinant human EGFR Fc chimera covering the sequence of the

2 extracellular domain of the protein, Met1-Ser645 (R\&D Systems) was injected simultaneously

3 over the EGF and the control surfaces at a series of concentrations (0 to $400 \mathrm{nM}$ ) with a flow rate

$45 \mu \mathrm{l} / \mathrm{min}$, for $7 \mathrm{~min}$, time during which binding reached a plateau in each case. The final sEGFR

5 binding sensogram was obtained by subtracting the bulk shift using the control sensogram. The

6 equilibrium binding constant $\mathrm{K}_{\mathrm{D}}$ was determined to be $11 \mathrm{nM}$ using the Biacore T100 Evaluation

7 software.

8 Competition assays were performed by injecting sEGFR with curcumin or free EGF on the

9 immobilized EGF in the same conditions. Mixtures were prepared 20 min prior to injection with a

10 final concentration of $600 \mathrm{nM}$ for sEGFR, $30 \mu \mathrm{M}$ for curcumin and $6 \mu \mathrm{M}$ for EGF. The surface

11 was regenerated between sEGFR injections with $5 \mathrm{mM} \mathrm{NaOH}$ during $1 \mathrm{~min}$ at $5 \mu \mathrm{l} / \mathrm{min}$.

\section{Measurement of lipid bilayer fluidity.}

13 Liposome formation and characterization. Lipids were dissolved in chloroform and mixed in a

14 glass vial with the fluorescent probes at the desired ratio. Chloroform was evaporated under gentle

15 nitrogen flow and the resulting film was stored in a dessicator in the dark and under vacuum for

16 minimum $2 \mathrm{~h}$ to remove organic solvent residues. The dry film was then rehydrated to a final lipid

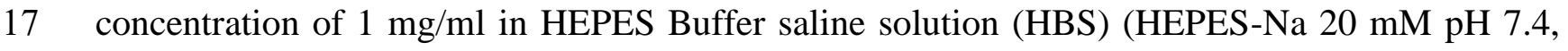

$18 \mathrm{NaCl} 150 \mathrm{mM}$ ) by $5 \mathrm{~min}$ vortexing. Vesicles were produced from the multilamellar vesicles

19 suspension by ultrasonic treatment ( 4 cycles of $3 \mathrm{~min}$ at $100 \mathrm{~W}$ each, separated by a $3 \mathrm{~min}$ pause)

20 during which the suspension was kept in an ice bath, with a tip sonicator (Ultrasonic Processor

21 Vibra Cell, Sonics Materials). The obtained suspension of small unilamellar vesicles (SUV) was

22 filtered immediately through a $0.2 \mu \mathrm{m}$ filter (Acrodisc). SUV size average and distribution were 
1 determined by dynamic light scattering (DLS) using a ZetaSizer Nano ZS (Malvern Instruments),

2 yielding a rather homogenous size with a diameter of $58 \pm 7 \mathrm{~nm}$.

3 Fluorescence anisotropy measurements. Steady-state fluorescence emission anisotropy $(r)$ was

4 measured with a Cary Eclipse Fluorescence Spectrophotometer (Agilent Technologies) equipped

5 with a thermostated cuvette holder. SUVs made of 1,2-dipalmitoyl-sn-glycero-3-phosphocholine

6 (DPPC, Sigma-Aldrich) and containing 1,6-diphenyl-1,3,5-hexatriene (DPH) or 1-(4-

7 trimethylammoniumphenyl)-6-phenyl-1,3,5-hexatriene $\quad$-toluenesulfonate (TMA-DPH)

8 fluorescent probes (Sigma-Aldrich) were diluted in HBS solution to the final concentration of 200

$9 \mu \mathrm{M}$ DPPC and $5 \mu \mathrm{M}$ fluorescent probe. Different volumes of curcumin stock solution in DMSO

$10(27 \mathrm{mM})$ were added directly in the quartz cuvette to obtain the desired final concentration and

11 were incubated with the SUVs for $30 \mathrm{~min}$ in the dark prior to fluorescence measurement.

12 Fluorescence intensities were collected at $435 \mathrm{~nm}$ for DPH and $428 \mathrm{~nm}$ for TMA-DPH with

13 excitation wavelengths 357 and 358 respectively. Anisotropy was automatically calculated by the

14 software of the spectrophotometer according to Equation 1:

$15 \quad r=\frac{I_{0}-G I_{90}}{I_{0}+2 G I_{90}}(1)$

16 where $I_{0}$ is the fluorescence intensity measured with polarizer in parallel orientation $\left(0^{\circ}\right)$ and $I_{90}$

17 the intensity in perpendicular orientation (excitation $0^{\circ}$ and emission $90^{\circ}$ ). $G$ is the correction factor

18 derived from the ratio of emission intensity at 0 and $90^{\circ}$ with the excitation polarizer at $90^{\circ}$ and is

19 taking into account the different sensitivity of the detection system for vertically and horizontally

20 polarized light (Equation 2):

$21 \quad G=\frac{I_{90}}{I_{0}}(2)$ 
3 Fluorescence Recovery After Photobleaching (FRAP). The diffusion coefficient of lipids was

4 determined using FRAP. Experiments were carried out in a confocal laser-scanning system (LSM

5 710, Carl Zeiss) based on an AxioObserver Z1 inverted microscope (Carl Zeiss). Observations

6 were done with a $63 \mathrm{x}$ immersion objective.

7 For fluorescence measurements, a lipid fluorescent probe (1,2-dipalmitoyl-sn-glycero-38 phosphoethanolamine-N-(7-nitro-2-1,3-benzoxadiazol-4-yl) triethylamine salt, DPPE-NBD 9 (Molecular Probes)) was added into the vesicles at a $2 \%$ molar ratio. Supported lipid bilayer (SLB)

10 formation was performed by deposition of EggPC/DPPE-NBD vesicle suspension ( $1 \mathrm{mg} / \mathrm{ml})$ into 11 a $400 \mu \mathrm{l}$ measurement cell, obtained from the top half of a spectrophotometer cuvette (internal 12 diameter $1 \mathrm{~cm}$ ) glued onto ultraclean glass cover slide. After 1 hour, the cell was carefully flushed 13 with HEPES buffer. Concentrated solution of curcumin pre-diluted in HEPES buffer was added 14 directly in the cell and incubated with the SLB 20 min prior each FRAP measurement.

15 In a typical experiment, the NBD fluorescent probe was excited by an argon ion laser (488 nm, 25

$16 \mathrm{~mW}$ ) and the emitted corresponding image (square of $225 \mu \mathrm{m} \times 225 \mu \mathrm{m}$ ) was collected by a 17 detector LSM-P-PMT (Carl Zeiss) during a 1 s scan. A smaller area (square of $45 \mu \mathrm{m}$ x $45 \mu \mathrm{m}$ ) 18 was bleached using full laser power (100\%) in scanning mode for $4 \mathrm{~s}$. The fluorescence recovery 19 was recorded with a low laser power (2\%). Images were acquired every $4 \mathrm{~s}$ via the integrated Zen 20 software (Carl Zeiss). Quantitative analysis of the gray levels of the bleached area was performed 21 to evaluate the fluorescence recovery using ImageJ software. Diffusion coefficient and the mobile 22 fraction of the lipids were determined from the fit of the experimental normalized fluorescence 
1 intensity recovery curve to the theoretical one established according to the calculations described

2 previously. ${ }^{34,35}$

3 Single Particle Tracking (SPT) experiments. Two days before the experiments, cells were plated

4 on coverslips. Streptavidin-coated Quantum Dots (QD) (655 nm, Q10121MP, Molecular Probes,

5 Life Technologies) were pre-coupled to a biotinylated anti-hEGFR antibody (R\&D systems) by a

615 min incubation in PBS with BSA $1 \%$ and D-glucose 2\% (PBS+) at a 1:10 molar ratio QD :

7 antibody. Prior to microscopy observations, the coverslips were mounted in a chamber $(25 \mathrm{~mm}$

8 inner diameter) and the EGF receptors were then labelled for $30 \mathrm{~min}$ with $0.03 \mathrm{nM}$ conjugated QD

9 in PBS+ $(100 \mu \mathrm{L})$. Unbound QD were then washed away by replacing the solution three times

$10 \quad$ with $3 \mathrm{~mL}$ PBS+.

11 Tracking of the QD at the cell surface was performed at room temperature $\left(22 \pm 1{ }^{\circ} \mathrm{C}\right)$ on an

12 Axioplan 2 microscope (Zeiss) equipped with a Cascade II 512 EM-CCD camera (Roper

13 Scientific) operating at $25 \mathrm{~Hz}$ acquisition frequency. QD were illuminated by means of an X CITE

14120 light source containing a metal halide vapour short arc lamp and observed through a Fluar

$15100 \mathrm{X} / 1.30$ oil UV objective associated to a $1.6 \mathrm{X}$ multiplier tube lens connected to the camera.

16 The duration of the recordings was set to $20 \mathrm{~s}$.

17 The trajectories of all QD of a video sequence were determined using the Mutiple Target Tracing

18 program developed by Sergé and collaborators. ${ }^{36}$ Only trajectories of more than $5 \mathrm{~s}$ were further

19 analyzed with a homemade program written in Visual Basic (VBA Excel, Microsoft) computing

20 the Mean Square Displacement as a function of time interval, $\operatorname{MSD}(n \Delta \mathrm{t})$, using the formula

21 (Equation 3):

$22 M S D(n \Delta t)=\frac{1}{N-n} \sum_{i=1}^{N-n}\left\{\left(x_{n+i}-x_{i}\right)^{2}+\left(y_{n+i}-y_{i}\right)^{2}\right\}$ (3) 
1 where $\mathrm{n}$ is the number of time intervals, $\Delta \mathrm{t}$ is the time interval between two successive frames (40

$2 \mathrm{~ms}), \mathrm{N}$ is the total number of frames, in our case $\mathrm{N} \leq 500$ frames, $\mathrm{x}(\mathrm{t})$ and $\mathrm{y}(\mathrm{t})$ are the QD

3 coordinates at time t.

4 To obtain the most accurate and precise values of the diffusion coefficients, we estimated the short

5 term diffusion coefficient $D_{1-2}$ obtained from only the first two $\operatorname{MSD}(n \Delta \mathrm{t})$ points: $D_{1-2}=$ $6 \quad[\operatorname{MSD}(2 \Delta t)-\operatorname{MSD}(\Delta t)] /(4 \Delta t)$ independently of the diffusion mode of the particles. ${ }^{37}$

7 Statistical analysis. All statistical evaluations were performed using GraphPad InStat software

8 and were based on Mann-Whitney $U$ test, where: *, $p \leq 0.05$, significant; **, $p \leq 0.01$, very

9 significant; $* * *, p \leq 0.001$, extremely significant; $* * * *, p \leq 0.0001$, extremely significant

10 (threshold and nomenclature as in GraphPad Software). The number of experiments is indicated 11 in each figure caption. 
2 Curcumin decreases the EGF induced phosphorylation level of EGFR in A431 cells.

3 We investigated the short-term effect of curcumin on the EGF-induced EGFR phosphorylation in

4 human epidermoid carcinoma cells (A431) at low polyphenol concentrations. The choice of this

5 cell line was motivated by the high expression level of EGFR, which was estimated to be up to 2-

$63 \times 10^{6}$ receptors per cell. ${ }^{38}$ The fluorescence signal due to the presence of phosphorylated EGFR

7 (Y1068) was measured in whole A431 cells and normalized to the fluorescence signal related to

8 the total EGFR (pan-protein) content using a cell-based immunoassay. The Y1068 residue belongs

9 to the group of the first tyrosine residues to be autophosphorylated. ${ }^{39}$ As the ligand incubation time

10 applied for phosphorylation studies was found to vary between 2 min and $1 \mathrm{~h}$ in the literature. ${ }^{40-42}$

11 We first examined the EGFR phosphorylation yield as a function of the time of cell exposure to

$12100 \mathrm{ng} / \mathrm{mL}$ EGF (Figure S1). The highest phosphorylation yield was obtained for $1 \mathrm{~h}$ with a 4-fold

13 increase compared to the basal level in the absence of EGF treatment. Therefore this exposure

14 duration was used in the following experiments and the EGF induced phosphorylation degree was

15 further taken as our reference to determine inhibition levels. Based on the previously published

16 results $^{25}$, curcumin pre-incubation time before EGF treatment was set to $4 \mathrm{~h}$. 


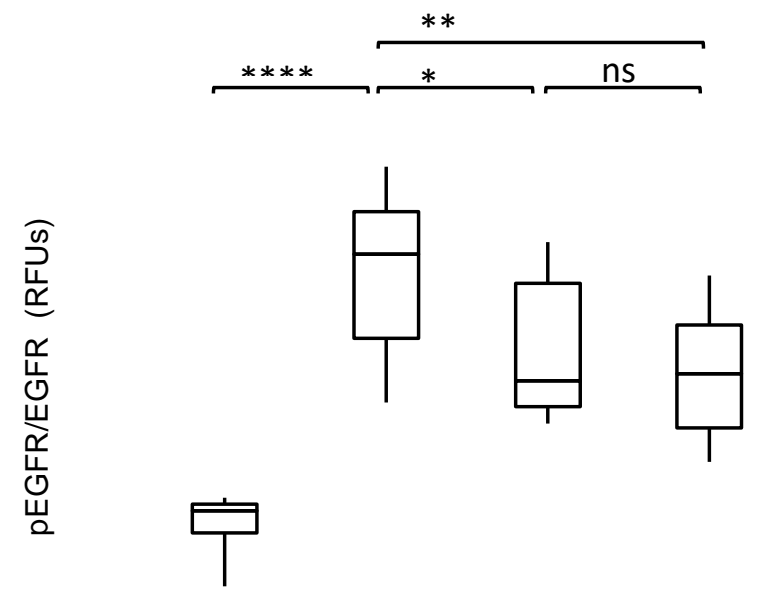

2 Figure 1. Box plot presenting EGF induced phosphorylation level of EGFR on Y1068 in A431 3 cells after treatment with assigned agents at specified concentrations added to serum free culture 4 media. The phosphorylation levels were normalized to the EGFR level and expressed in 5 normalized relative fluorescence units (RFU). Whiskers: from min to max values $(\mathrm{n}=8)$. Asterisks 6 indicate significant differences in phosphorylation levels in treated and control cells (Mann$7 \quad$ Whitney $U$ test): *,$p \leq 0.05 ; * *, p \leq 0.01$; ****; $p \leq 0.0001$.

9 Data presented in the Figure 1 show that curcumin 4 h-treatement leads to a decrease in the EGF 10 induced phosphorylation of EGFR. Significant differences were obtained starting $1 \mu \mathrm{M}$ of 11 curcumin under our experimental conditions. The average phosphorylation level was reduced by $1240 \%$ in comparison to the EGF-activated reference after exposure of the cells to $5 \mu \mathrm{M}$ curcumin.

13 In order to get a deeper insight into the mechanism by which the molecule is able to inhibit 14 phosphorylation of the Y1068 site on EGFR, additional experiments on the EGFR extra and intra 15 cellular domains were designed. 


\section{Curcumin does not affect EGF binding to EGFR extracellular domain.}

2 One hypothesis for curcuma action on ligand induced activation of EGFR is that this polyphenol

3 interfers in the ligand binding to the extracellular domain that enhances dimer stability and as a

4 consequence promotes trans-autophosphorylation. We used surface plasmon resonance

5 spectroscopy to investigate the ability of curcumin to compete with ligand binding to EGFR. EGF

6 was immobilized on a BIAcore CM5 sensor chip. The immobilization level of EGF yielded $295 \pm$

$754 \mathrm{RU}$ (three independent immobilizations) which is in the range of previously published data. ${ }^{43}$

8 The soluble extracellular domain of EGFR (sEGFR) was passed at different concentrations over

9 these surfaces and the maximum binding response (Rmax) was plotted against sEGFR

10 concentration (Figure S2). The best fit of the generated binding curve was obtained using a two-

11 sites Langmuir model. We chose this model since it yielded a much better fit than the one-site

12 model, and because sEGFR from human origin does not dimerize in solution, thus ruling out the

13 cooperativity (Hill) model that has been suggested for sEGFR from other species. ${ }^{44}$ The fit leads

14 to the determination of two equilibrium constants $\mathrm{K}_{\mathrm{D} 1}=0.97 \pm 0.10 \mathrm{nM}$ and $\mathrm{K}_{\mathrm{D} 2}=60.76 \pm 5.17$

$15 \mathrm{nM}$ with equivalent maximum number of binding sites (BMAX1: $109.0 \pm 4.3$ and $\mathrm{B}_{\mathrm{MAX} 2:} 166.2 \pm$

163.8 , respectively). These two affinity levels for EGF may be due to different conformations that

17 sEGFR can adopt in solution. There are two populations of EGFR regarding affinity towards

18 binding EGF correlated with the conformational changes of the receptor. ${ }^{45-48}$ However this does

19 not constitute a limitation for our competition studies since in both case sEGFR bound strongly to

20 the immobilized EGF and the $\mathrm{K}_{\mathrm{D}}$ are low enough for competitive assays.

21 Samples of $600 \mathrm{nM}$ of sEGFR containing increasing concentrations of curcumin were injected

22 over the EGF-derivatized sensor surface. No influence on the equilibrium SPR response of sEGFR

23 binding could be observed even with up to a 50-fold molar excess of curcumin (Figure 2). A 
1 positive control was performed using a 1:10 mixture of sEGFR and free EGF, the binding of

2 sEGFR to immobilized EGF was fully blocked in this case. This results shows that curcumin does

3 not act as a ligand binding inhibitor.

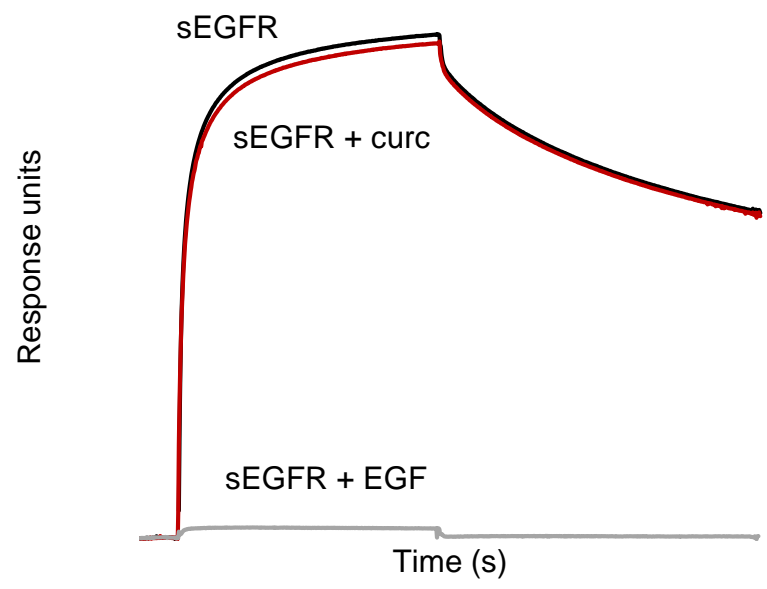

4

5 Figure 2. Surface Plasmon resonance competition analysis showing the effect of curcumin (30 $6 \mu \mathrm{M})$ and free EGF $(6 \mu \mathrm{M})$ upon the binding of $600 \mathrm{nM}$ sEGFR to immobilized EGF. Mixture of 7 sEGFR and curcumin or ligand.

\section{Inhibitory effect of curcumin on EGFR tyrosine kinase domain activity.}

10 The tyrosine kinase inhibitory effect of curcumin on a recombinant, 695-end, active human EGFR

11 domain (EGFR-TK) was evaluated (Figure 3) using a universal tyrosine kinase assay. The selected

12 EGFR intracellular domain concentration of $141 \mathrm{pM}$ leads to a tyrosine kinase activity of $22.6 \mathrm{x}$

$1310^{-5} \mathrm{U} / \mu \mathrm{l}$ which is in the sensitivity range of the kit. Curcumin was incubated at different final

14 concentrations (1 to $5 \mu \mathrm{M}$ ) with the protein before initiating the phosphorylation reaction by ATP

15 addition. A partial inhibition of the tyrosine kinase activity of EGFR intracellular domain was

16 observed for the whole range of concentration of curcumin considered. 


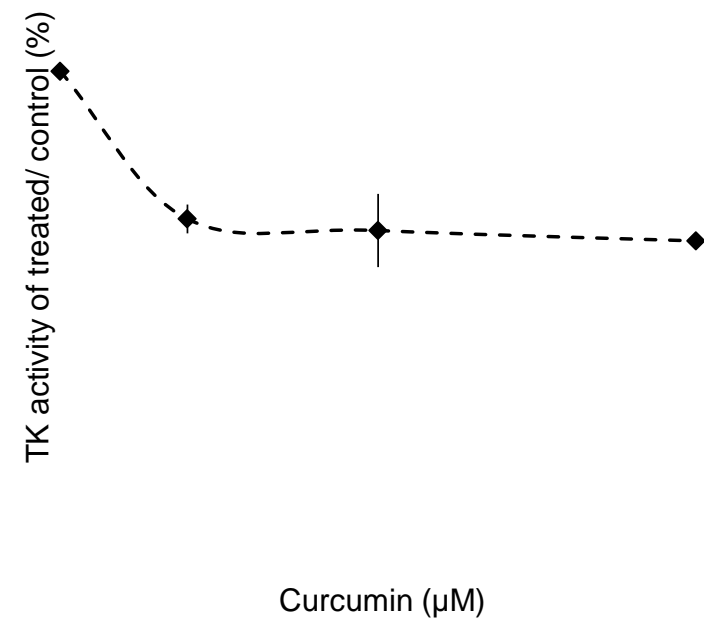

2 Figure 3. Inhibitory effect on EGFR tyrosine kinase domain by curcumin. The results were 3 normalized by dividing the tyrosine kinase activity of EGFR-TK in the presence of curcumin by 4 the one measured in its absence. Average and standard deviations were calculated from 3 5 independent experiments.

7 The inhibition at $1 \mu \mathrm{M}$ curcumin was equal to $26.8 \pm 2.6 \%$. This effect increased slightly with the 8 augmentation of the curcumin concentration and reached $30.8 \pm 0.9 \%$ at $5 \mu \mathrm{M}$. A partial inhibition 9 of the EGFR kinase had already been observed with other inhibitor compounds using life cell 10 kinase assays ${ }^{49}$ and was confirmed for curcumin by our results with A431 cells in Figure 1. To 11 speculate on a potential reason for this partial inhibition of EGFR-TK, the above observation of 12 high and low affinity binding of EGF to EGFR, leading us to postulate two populations of sEGFR, 13 might in turn indicate the existence of two possible conformations of the whole protein and thus 14 also of the EGFR-TK domain, with different affinities for the inhibitor.

15 Curcumin is thus able to act on the EGFR phosphorylation via a direct action on the tyrosine kinase 16 domain as expected for a small and hydrophobic molecule. Nevertheless, the inhibition of tyrosine 17 kinase activity by curcumin observed here appears to be slightly lesser than the inhibition of EGFR 
1 phosphorylation on the Y1068 site determined in A431 cells (Figure 1) which reached an average

2 of $40 \%$ for $5 \mu \mathrm{M}$ of curcumin. This is all the more surprising given that in the TK assays the

3 experiment was performed in an environment deprived of other factors than the tested agent, while

4 in cells the total curcumin concentration is not available for interacting with the TK domain since

5 it is located in different membrane compartments, i.e. incorporated into plasma membrane or

6 nucleus. ${ }^{50}$ This led us to consider the involvement of an additional mechanism in curcumin

7 mediated EGFR inhibition at the cellular level. Curcumin is known to interact with the plasma

8 membrane. ${ }^{31,32,51}$ The membrane is the natural environment of EGFR and plays an important role

9 in the receptor diffusion, which in turn is essential for its dimerization resulting in activation. The

10 question was therefore whether curcumin could affect the membrane properties to an extent that

11 would alter the EGFR lateral diffusion? To answer this question, analyses allowing to better

12 understand the curcumin effect on the membrane fluidity were performed.

\section{Curcumin decreases the lipid bilayer fluidity}

15 Local membrane viscosity changes were assessed by steady-state fluorescence anisotropy studies

16 providing a sensitive indicator of the motional freedom of a fluorescent probe inserted in a

17 membrane. ${ }^{52}$ The partitioning of two fluorescent probes, TMA-DPH and DPH, has been employed

18 to monitor the influence of curcumin on the lipid dynamics properties in different membrane

19 regions. The polar region of TMA-DPH is anchored at the lipid-water interface and DPH locates

20 in the hydrophobic core of the lipid bilayer. ${ }^{53}$ The fluorescence anisotropy values are inversely

21 proportional to membrane fluidity in the probe environment. ${ }^{54} \mathrm{We}$ performed the anisotropy

22 measurements on pure DPPC vesicles. This strategy allowed us to determine the influence of 
1 curcumin on lipid fluidity at two different gel/liquid-crystalline phospholipid order, as DPPC has

2 a phase transition at about $41.5^{\circ} \mathrm{C}$. The effect of curcumin on the local fluidity along graded depths 3 in the phospholipid bilayer is presented Figure 4.
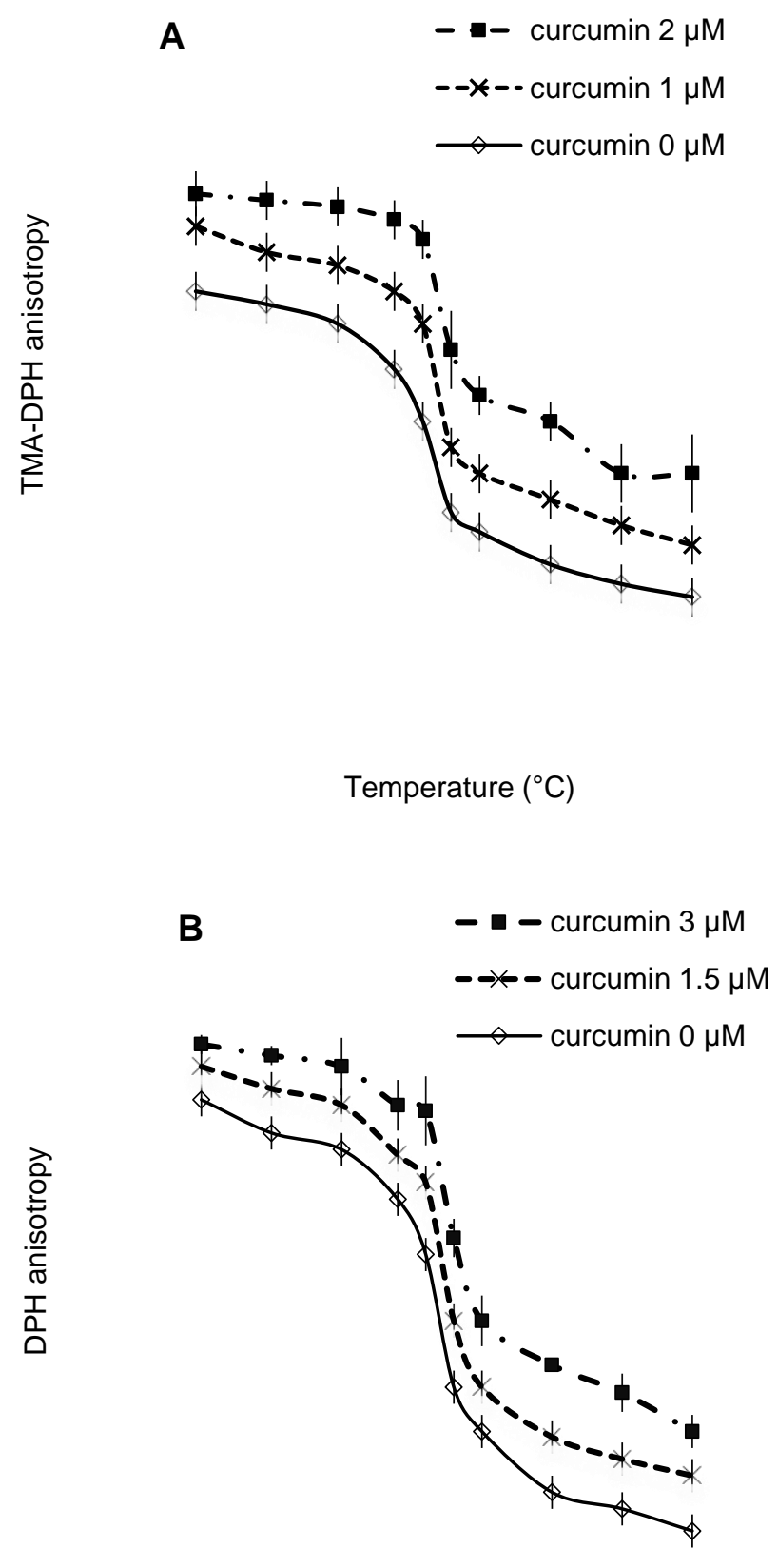
1 Figure 4. Average values of steady-state fluorescence anisotropy for TMA-DPH (A) and DPH (B) 2 probes in DPPC vesicles $(200 \mu \mathrm{M})$ with different concentration of curcumin $(1$ to $3 \mu \mathrm{M})$ indicated 3 in the legend in the temperature range. Averages were calculated from 3 independent experiments. 4 For clarity, only the anisotropy plots corresponding to curcumin concentrations that lead to 5 significant anisotropy changes are shown.

7 The anisotropy values were increasing with increasing curcumin concentrations over the whole temperature range for both fluorescent probes. This rise was observed in both the solid gel and

9 liquid-crystalline phases, as well as around the melting transition. These results demonstrate a 10 strong influence of curcumin on the fluidity of the lipid bilayer both in the interfacial layer and in

11 the hydrophobic core. Interestingly, the critical concentration of curcumin at which the first 12 significant changes appear was lower for TMA-DPH $(1 \mu \mathrm{M})$ than for DPH $(1.5 \mu \mathrm{M})$. If we 13 consider the location of these probes in the lipid bilayer, we can assume that the external leaflet 14 was more sensitive to the curcumin triggered changes than the inner hydrophobic compartment. 15 That implies that the molecule is gradually embedded into membrane structure, at low 16 concentration it stands exclusively in the interface and when the concentration increases, it enters 17 deeper into the hydrophobic core. Such a mechanism of the interaction of the curcumin with the 18 membrane has been already described by Barry and collaborators. ${ }^{55}$

19 Furthermore, the curcumin effect on the lateral diffusion of phospholipids in the lipid bilayer was 20 quantified using the fluorescence recovery after photobleaching (FRAP) technique. This assay was

21 performed on an EggPC/NBD-DPPE bilayer supported on a glass surface (SLB). The florescence 22 recovery of a bleached area of the SLB containing fluorescent lipids was monitored after 23 incubation with increasing concentrations of curcumin added in the buffer reservoir above the 24 bilayer. The lipid diffusion coefficient $(D)$ was plotted as a function of curcumin concentration in 25 Figure 5. The value of the lateral coefficient in the absence of lipids is consistent with the 
1 previously published data for SLB of the same lipid composition. ${ }^{56,57}$ Curcumin significantly

2 decreases lipid lateral diffusion at concentration above $0.4 \mu \mathrm{M}$.

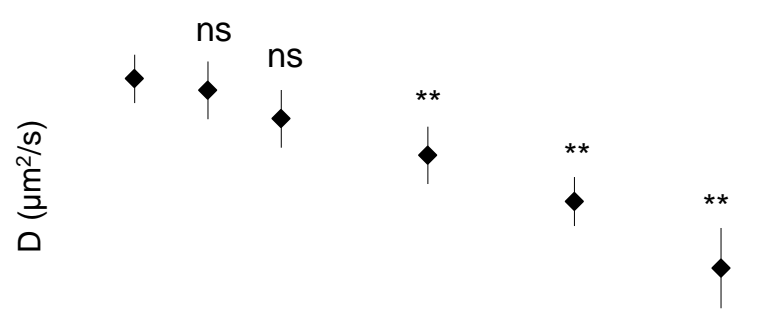

Curcumin $(\mu \mathrm{M})$

5 Figure 5. Lateral diffusion coefficient of NDB-DPPE in EggPC SLB upon treatment with 6 indicated concentration of the curcumin $(n=6)$. Asterisks indicate significant differences in 7 diffusion coefficients obtained in the presence and in the absence of curcumin (Mann-Whitney $U$ 8 test): ${ }^{* *}, p \leq 0.01$.

10 Together with the fluorescence anisotropy data, these findings confirm the proposed scenario of

11 the rigidification of the membrane by curcumin. In membrane models, this effect is significant

12 already at low curcumin concentrations. Therefore one may conclude that it should also impact the

13 diffusion of the membrane proteins. Nevertheless, all of the aforementioned tests were conducted

14 in biomimetic membrane models. These structures, despite having several advantages for practical

15 reasons, do not exactly mirror the complex cell membrane properties. In order to confirm our

16 hypothesis that receptor diffusion is impacted by the membrane rigidification caused by curcumin,

17 we studied the alteration of EGFR mobility by curcumin in the plasma membrane of A431 cells in

18 single particle tracking experiments. In fact, the EGFR diffusion coefficient is in the $10^{-2} \mu \mathrm{m}^{2} / \mathrm{s}$ 
1 range ${ }^{58,59}$, which is at the limit of detection of FRAP. ${ }^{60}$ The evaluation of its decrease caused by

2 curcumin required thus a more sensitive technique.

3 Single particle tracking of quantum dot labelled EGFR in the plasma membrane of A431

$4 \quad$ cells treated with curcumin

5 We employed the single particle tracking technique to measure the diffusion coefficient of the

6 EGFR in the A431 cells treated or not with curcumin. The conditions of cells treatment were

7 exactly the same as those applied for studies of the EGFR phosphorylation level in cells.

8 Functionalisation of quantum dots (QD) was optimised in a two-step procedure. Firstly, binding

9 affinity of biotinylated EGFR antibodies purchased from three different suppliers were tested to

10 choose the one with the highest affinity. Secondly, QD conjugated to Ab at different ratios were

11 prepared and 20 short trajectories of $5 \mathrm{~s}$ acquired to rapidly evaluate the diffusion coefficient. The

12 best condition was chosen as the one giving the highest value of the diffusion coefficient,

13 corresponding to a QD:Ab molar ratio of $1: 10 .{ }^{37}$ Prior to SPT experiments, the specificity of the

14 labelling was verified by counting the average number of QD bound per cell after incubation of

15 the cells with QD conjugated to EGFR antibodies in comparison to bare QD. The fraction of

16 unspecific binding was found to be $6.5 \%$.

17 SPT data show variations in the short term diffusion coefficient $D_{1-2}$ obtained in the different

18 conditions. EGF addition to the cells starved in serum-free medium prior to the measurements

19 caused significant elevation of the average diffusion coefficient of the EGFR, $D_{1-2}=0.034 \pm 0.002$

$20 \mu \mathrm{m}^{2} \mathrm{~s}^{-1}$, compared to that measured in cells not stimulated with the ligand, $D_{1-2}=0.025 \pm 0.002$

$21 \mu \mathrm{m}^{2} \mathrm{~s}^{-1}$. Curcumin addition to the cells medium before stimulation with the ligand reduced the EGF

22 effect. The presence of the polyphenol at $1 \mu \mathrm{M}$ in the sample pre-treated with the ligand did not 
1 affect significantly the measured diffusion coefficient, but at $5 \mu \mathrm{M}$ curcumin the mean $D_{1-2}=0.025$

$2 \pm 0.001 \mu \mathrm{m}^{2} \mathrm{~s}^{-1}$, was reduced to its value in non-stimulated cells (Figure 6 and S3).

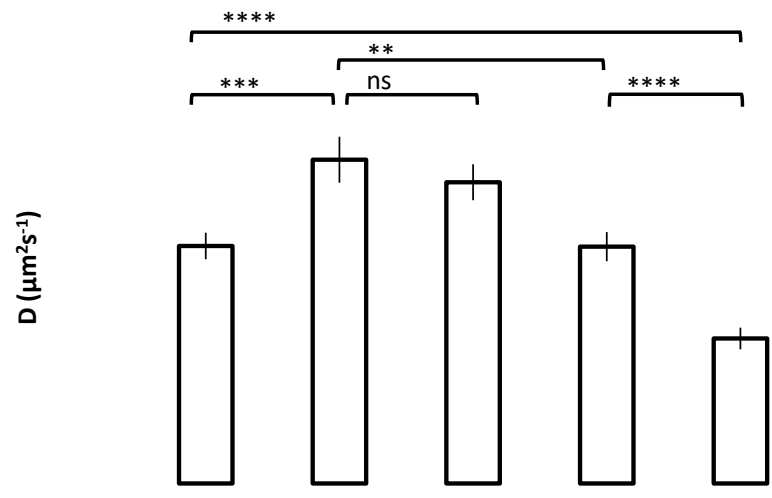

$4 \quad$ Figure 6. Mean diffusion coefficient determined by SPT for the EGFR labelled with QD in A431 5 cells cultured in SFM for $24 \mathrm{~h}$ prior to measurement and treated with indicated solutions, curcumin 6 (curc) was introduced $4 \mathrm{~h}$ before the measurement, EGF at $100 \mathrm{ng} / \mathrm{ml}$ was added in parallel with 7 QD-Ab; bars represent the standard error; $109 \leq n \leq 173$. Asterisks indicate significant differences 8 in EGFR diffusion coefficients compared to the control condition (EGF treatment) (Mann-Whitney $9 \quad U$ test): **, $p \leq 0.01 ; * * *, p \leq 0.001 ; * * * *, p \leq 0.0001$.

11 Once established that curcumin affects the diffusion coefficient of the ligand activated EGFR in 12 A431 cells, we further tested if the effect was depending of the EGF-mediated activation of the 13 receptor. For that, the experiment was repeated for $5 \mu \mathrm{M}$ curcumin without the addition of EGF.

14 As presented in Figure 6, the mean value of $D_{1-2}$, compared to the one in the absence of curcumin

15 (and EGF) also decreased in this case $\left(D_{1-2}=0.015 \pm 0.001 \mu \mathrm{m}^{2} \mathrm{~s}^{-1}\right)$. These findings suggest that 16 curcumin reduces the EGFR diffusion in A431 plasma membrane independently of the ligand 17 induced receptor activation. 
1 DISCUSSION

2

3 In the present work, we aimed to investigate the mode of action of curcumin on the EGFR

4 phosphorylation. We first evaluated the effect of curcumin on EGFR autophosphorylation in

5 human epithelial cancer cells (A431). The curcumin treatment of A431 was already found to affect

6 the autophosphorylation activity of the EGFR tyrosine kinase in a dose- and time- dependent

7 manner. ${ }^{25}$ In the cited study, the highest inhibition level was obtained for a $4 \mathrm{~h}$ pre-treatment of

8 cells, and the lowest concentration tested was $10 \mu \mathrm{M}$. Most of the studies being conducted on the

9 inhibition of EGFR kinase activity by curcumin in cells have been performed using high

10 concentrations of curcumin up to $5 \mu \mathrm{M} .{ }^{24,61-63}$ However, such concentrations cannot be achieved

11 by oral administration. Due to its poor bioavailability, the curcumin levels found in human serum

12 are very low, mostly in the $10-100 \mathrm{nM}$ range ${ }^{64}$; the highest value reported was $1.77+/-1.87 \mu \mathrm{M}$

13 after taking $8 \mathrm{~g}$ of curcumin. ${ }^{65}$ For these reasons, we restricted the range of the curcumin

14 concentrations applied on cells and membranes models to up to $5 \mu \mathrm{M}$.

15 The results obtained on A431 cells indicate that short-term treatment with curcumin affects the

16 phosphorylation of the Y1068 residue of EGFR. Curcumin significantly inhibits the EGF-mediated

17 tyrosine kinase activity starting at $1 \mu \mathrm{M}$ concentration after 4 hours of cell exposure to curcumin.

18 To our knowledge, this is the first time that an effect at such a low dose is reported. As Korutla

19 and Kumar already indicated, the early effect of curcumin on EGFR phosphorylation suggests a

20 direct action of curcumin on the membrane receptor at the molecular level. The author's

21 assumption is that curcumin may interfere with the ligand induced activation of EGFR. ${ }^{25}$ The

22 molecular mechanism of the short-term inhibition of EGFR kinase activity by curcumin remains

23 however unknown. 
1 To elucidate the mechanism by which curcumin directly acts on EGFR, we performed in vitro

2 experiments on separated recombinant EGFR domains. Surface competition assays were carried

3 out in order to determine the ability of curcumin to compete the soluble EGFR extracellular domain

4 binding to immobilized EGF. No effect on the SPR response of sEGFR binding to immobilized

5 EGF could be observed even for concentrations corresponding to a 50-fold molar excess of

6 curcumin. ELISA-based assays were employed to investigate the inhibitory effect of curcumin on

7 tyrosine kinase activity of EGFR intracellular domain. ${ }^{66}$ The results demonstrate that curcumin at

$85 \mu \mathrm{M}$ significantly lowered the EGFR-TK activity by $30 \%$. Thus, the direct action of curcumin

9 on the receptor is on its intracellular domain.

10 However, these in vitro experiments on soluble EGFR domains reflect only partially the molecular

11 mechanism of the short-term action of curcumin in cells. Like the majority of the tyrosine kinase

12 inhibitors, curcumin is a highly hydrophobic molecule with a $\log \mathrm{P}$ of 3.28 , causing its

13 accumulation in tissues and more specifically in the cellular membranes. ${ }^{32,}{ }^{67-70}$ The higher

14 curcumin inhibitory level in cellulo than in solution indicates that an additional molecular

15 mechanism may be involved in the early effect of curcumin on EGFR phosphorylation, related to

16 an accumulation of the polyphenol in the membrane. Considering that curcumin has been

17 suggested to change the properties of the cell membrane and to affect membrane-bound proteins

18 in an indirect manner ${ }^{71}$, we hypothesized that EGF receptor autophosphorylation was also

19 impacted by modulation of the membrane physical properties resulting from the curcumin

20 interaction with the EGFR surrounding.

21 As a first-line study, we investigated the effect of curcumin on the lipid bilayer fluidity in

22 biomimetic models. Cell membranes are composed of a lipid bilayer intercalated with other

23 constituents such as proteins. The complexity of native membranes and their interactions with the 
1 intra- and extracellular environment makes the precise effect of the studied polyphenol on

2 membrane properties difficult to track and to interpret. ${ }^{72}$ As an illustration, although a decrease in

3 the lateral mobility of lipids in membranes had already been reported for human cells treated with

$410 \mu \mathrm{M}$ of curcumin, this could not be clearly linked to the physical insertion of curcumin in

5 membrane and was attributed to the induction by curcumin of reactive oxygen species

6 production..$^{73}$ In this study, liposomes and planar lipid bilayers with different lipid compositions

7 were employed to mimic the organization of the cell membrane matrix in a simplified and

8 controlled manner. The measurements of TMA-DPH and DPH fluorescence anisotropy indicated

9 that curcumin affects the bilayer fluidity in the region where the fluorescent probes were located.

10 A significant fluidity decrease was observed at the interphase between the hydrophilic and the

11 hydrophobic parts of the bilayer starting $1 \mu \mathrm{M}$ and in the hydrophobic region starting $1.5 \mu \mathrm{M}$,

12 independent of the state of the lipid phase. The interaction mode of curcumin with the lipid bilayer

13 has been described to be a two-step process. ${ }^{74,75}$ Our results are consistent with the concentration

14 threshold of $1.5 \mu \mathrm{M}$, at which curcumin begins to intercalate into the bilayer's hydrophobic core,

15 as found by Chen and collaborators. The lateral fluidity of lipids in planar bilayer models is also

16 strongly decreased by curcumin insertion. First significant changes occur starting at $0.4 \mu \mathrm{M}$ of

17 curcumin. As FRAP analyses were performed on supported lipid bilayers and not on liposomes, a

18 parallel regarding the curcumin concentration threshold could not rigorously be drawn. Moreover,

19 the relative fragility of the lipid assembly in the SLB model may lead to amplified curcumin

20 effects. All together these results allow us to conclude that curcumin, at lower $\mu \mathrm{M}$ concentrations,

21 causes a pronounced dose-dependent rigidifying effect on the lipid bilayer, which is only based on

22 its insertion in the bilayer and which strongly affects the lateral mobility of the lipids. We can

23 logically assume that by modifying the membrane fluidity properties, curcumin may affect the 
1 spatiotemporal dynamic of EGFR in the membrane and, as a consequence, its activation resulting

2 from dimer formation.

3 In order to confirm the possible curcumin impact on EGFR mobility in the cell membrane,

4 the motion of single EGF receptors, labeled with quantum dots, was tracked in the membrane of 5 curcumin-treated A431 cells. Starting $5 \mu \mathrm{M}$ concentration, curcumin decreases significantly the 6 average diffusion coefficient of EGFR in the plasma membrane of A431 cells, confirming our

7 hypothesis that the molecular mechanism of curcumin inhibition of EGFR autophosphorylation is

8 dual and combines an action on the tyrosine kinase activity together with an effect on the EGFR

9 lateral mobility in the membrane. It is known that EGFR dimerization and its resulting

10 autophosphorylation is strongly dependent on the receptor diffusion governed by the fluidity of its

11 surrounding membrane. This influence of membrane fluidity on the receptor dimerization and its

12 consecutive activation was already demonstrated by studying the influence of the cholesterol

13 content in membrane models and plasma membranes. ${ }^{76,}{ }^{77}$ Cholesterol depletion from the

14 membrane results in enhanced EGFR dimerization and causes an increase in receptor

15 autophosphorylation, and vice versa. The cholesterol effect on EGFR activity is rather related to

16 the physical effects on membrane properties than direct interactions with the receptor. ${ }^{78}$ However,

17 the comparison of the action of curcumin on fluidity and the cholesterol content cannot be further

18 extended since cholesterol plays a complex role, not yet completely elucidated, in EGFR clustering

19 in cholesterol and phosphatidic acid-enriched nanodomains. ${ }^{76,79,80}$ The effect of curcumin to slow

20 down the lateral movement of EGFR in the plane of the membrane was observed upon ligand

21 stimulation as well as upon ligand-independent activation. This result indicates that the action of

22 curcumin on the membrane is not specifically related to the organization of EGFR in nanoclusters, 
1 which is driven by changes in lipid properties in response to EGF-induced activation of the 2 receptor..$^{59,79}$

\section{CONCLUSION}

The main goal of this study was to improve our understanding of the mode of action of curcumin

11 in EGFR inhibition. We demonstrated that the overall short-term inhibition of EGFR

12 phosphorylation by curcumin, observed in A431 cells, is a combined effect of the direct curcumin

13 action on tyrosine kinase domain and alterations of the physical plasma membrane properties

14 influencing receptor dimerization. Indeed, in addition to the direct inhibition of the enzymatic

15 activity, we identified an additional mechanism based on the curcumin interaction and

16 accumulation in the plasma membrane. Transposing the curcumin concentration range employed

17 in our study to human organism relies on higher local curcumin concentration in membranes than

18 in plasma. A contribution of the membrane in the mode of action of curcumin on EGFR allows to

19 better take into account the extremely poor solubility of curcumin in plasma and cytosol. Curcumin

20 insertion into the membrane leads to a lipid bilayer rigidification and a receptor lateral mobility

21 decrease, which is correlated to receptor dimerization and modulation of autophosphorylation. By

22 this dual mode of action, curcumin is able to affect receptor activity in cells at low micromolar

23 concentrations. Curcumin therefore appears to be a promising compound that may act on both

24 ligand-induced and ligand-independent EGFR activation and their related cancer types. Beyond

25 EGFR, the results of this study highlight the importance of the interaction of active molecules with 26 membranes in cancer treatment. 
ASSOCIATED CONTENT

3 Supporting Information. Phosphorylation level of EGFR on Y1068 in A431 cells as a function

4 of EGF exposure time. Surface Plasmon Resonance analysis of curcumin inhibition of the sEGFR

5 binding to immobilized EGF. Frequency histograms of D (log scale), the diffusion coefficient of

6 EGFR as determined from SPT measurements on the A431 cells treated with curcumin. This

7 material is available free of charge via the Internet at http://pubs.acs.org.

\section{AUTHOR INFORMATION}

\section{Corresponding Author}

$12 *$ Tel.: 00333442345 85. E-mail: claire.rossi@utc.fr.

\section{Notes}

14 The authors declare no competing financial interest.

\section{ACKNOWLEDGMENT}

17 This research was supported by grants from the Centre National de la Recherche Scientifique 18 (CNRS), the European Union (co-funding of equipment within the CPER 2007-2013, and Marie

19 Curie Research Training Network CHEBANA, FP7 ITN 2010-264772). We thank F. Oudet and

20 all his collaborators for their help and advices. We thank Pascale Vigneron for her precious 21 technical assistance. 
(1) Schlessinger, J. Cell 2000, 103, 211-25.

(2) Ullrich, A.; Schlessinger, J. Cell 1990, 61, 203-12.

(3) Yarden, Y.; Sliwkowski, M. X. Nat. Rev. Mol. Cell Biol. 2001, 2, 127-37.

(4) Yarden, Y.; Ullich, A. Annu. Rev. Biochem. 1988, 57, 443-78.

(5) Bublil, E. M.; Yarden, Y. Curr. Opin. Cell Biol. 2007, 19, 124-34.

(6) Daley, G. Q.; Van Etten, R. A.; Baltimore, D. Science 1990, 247, 824-30.

(7) Mendelsohn, J.; Baselga, J. Oncogene 2000, 19, 6550-65.

(8) Garrett, T. P.; McKern, N. M.; Lou, M.; Elleman, T. C.; Adams, T. E.; Lovrecz, G. O.; Zhu, H. J.; Walker, F.; Frenkel, M. J.; Hoyne, P. A.; Jorissen, R. N.; Nice, E. C.; Burgess, A. W.; Ward, C. W. Cell 2002, 110, 763-73.

(9) Lemmon, M. A.; Bu, Z.; Ladbury, J. E.; Zhou, M.; Pinchasi, D.; Lax, I.; Engelman, D. M.; Schlessinger, J. EMBO J 1997, 16, 281-94.

(10) Ogiso, H.; Ishitani, R.; Nureki, O.; Fukai, S.; Yamanaka, M.; Kim, J. H.; Saito, K.; Sakamoto, A.; Inoue, M.; Shirouzu, M.; Yokoyama, S. Cell 2002, 110, 775-87.

(11) Olayioye, M. A.; Neve, R. M.; Lane, H. A.; Hynes, N. E. EMBO J. 2000, 19, 3159-67.

(12) Sako, Y.; Minoghchi, S.; Yanagida, T. Nat. Cell Biol. 2000, 2, 168-72.

(13) Klinger, B.; Sieber, A.; Fritsche-Guenther, R.; Witzel, F.; Berry, L.; Schumacher, D.; Yan, Y.; Durek, P.; Merchant, M.; Schafer, R.; Sers, C.; Bluthgen, Mol. Syst. Biol. 2013, 9, 673.

(14) Roskoski, R. Jr. Pharmacol. Res. 2014, 87C, 42-59.

(15) Chen, X.; Zhu, Q.; Zhu, L.; Pei, D.; Liu, Y.; Yin, Y.; Schuler, M.; Shu, Y. Lung Cancer 2013, $81,155-61$.

(16) Mendelsohn, J. J. Steroid Biochem. Mol. Biol. 1990, 37, 889-92.

(17) Ward, W. H.; Cook, P. N.; Slater, A. M.; Davies, D. H.; Holdgate, G. A.; Green, L. R. Biochem. Pharmacol. 1994, 48, 659-66.

(18) Chong, C. R.; Janne, P. A. Nat. Med. 2013, 19, 1389-400.

(19) Yewale, C.; Baradia, D.; Vhora, I.; Patil, S.; Misra, A. Biomaterials 2013, 34, 8690-707.

(20) Jung, S. K.; Lee, M. H.; Lim, D. Y.; Lee, S. Y.; Jeong, C. H.; Kim, J. E.; Lim, T. G.; Chen, H.; Bode, A. M.; Lee, H. J.; Lee, K. W.; Dong, Z. Mol. Carcinog. 2014. DOI: 10.1002/mc.22191

(21) Lin, J. K.; Lin-Shiau, S. Y. Mol. Nutr. Food Res. 2006, 50, 211-7.

(22) Chen, A.; Xu, J.; Johnson, A. C. Oncogene 2006, 25, 278-287.

(23) Doumiati, S.; Haupt, K.; Rossi, C. J. Mol. Recognit. 2012, 25, 623-9.

(24) Korutla, L.; Cheung, J. Y.; Mendelsohn, J.; Kumar, R. Carcinogenesis 1995, 16, 1741-5.

(25) Korutla, L.; Kumar, R. Biochim. Biophys. Acta 1994, 1224, 597-600.

(26) Soung, Y. H.; Chung, J. Mol. Cancer Ther. 2011, 10, 883-91.

(27) Anand, P.; Kunnumakkara, A. B.; Newman, R. A.; Aggarwal, B. B. Mol. Pharm. 2007, 4, 807-18.

(28) Gupta, S. C.; Patchva, S.; Koh, W.; Aggarwal, B. B. Clin. Exp. Pharmacol. Physiol. 2012, 39, 283-99.

(29) Prasad, S.; Gupta, S. C.; Tyagi, A. K.; Aggarwal, B. B. Biotechnol. Adv. 2014, 32, 105364.

(30) Somers-Edgar, T. J.; Scandlyn, M. J.; Stuart, E. C.; Le Nedelec, M. J.; Valentine, S. P.; Rosengren, R. J. Int. J. Cancer 2008, 122, 1966-71.

45 (31) Jaruga, E.; Salvioli, S.; Dobrucki, J.; Chrul, S.; Bandorowicz-Pikula, J.; Sikora, E.; 46 Franceschi, C.; Cossarizza, A.; Bartosz, G. FEBS Lett. 1998, 433, 287-93. 
(32) Jaruga, E.; Sokal, A.; Chrul, S.; Bartosz, G. Exp. Cell Res. 1998, 245, 303-12.

(33) Ji, C.; Cao, C.; Lu, S.; Kivlin, R.; Amaral, A.; Kouttab, N.; Yang, H.; Chu, W.; Bi, Z.; Di, W.; Wan, Y. Cancer Chemother. Pharmacol. 2008, 62, 857-65.

(34) Rossi, C.; Briand, E.; Parot, P.; Odorico, M.; Chopineau, J. J. Phys. Chem. B 2007, 111, 7567-76.

(35) Rossi, C.; Homand, J.; Bauche, C.; Hamdi, H.; Ladant, D.; Chopineau, J. Biochemistry 2003, 42, 15273-83.

(36) Serge, A.; Bertaux, N.; Rigneault, H.; Marguet, D. Nat. Methods 2008, 5, 687-94.

(37) Mascalchi, P.; Haanappel, E.; Carayon, C.; Mazeres, S.; Salome, L. Soft Matter 2012, 8, 4462-4470.

(38) Haigler, H.; Ash, J. F.; Singer, S. J.; Cohen, S. Proc. Natl. Acad. Sci. U S A 1978, 75, 331721.

(39) Kim, Y.; Li, Z.; Apetri, M.; Luo, B.; Settleman, J. E.; Anderson, K. S. Biochemistry 2012, $51,5212-22$.

(40) Liang, Y. C.; Lin-shiau, S. Y.; Chen, C. F.; Lin, J. K. J. Cell Biochem. 1997, 67, 55-65.

(41) Pastore, S.; Lulli, D.; Fidanza, P.; Potapovich, A. I.; Kostyuk, V. A.; De Luca, C.; Mikhal'chik, E.; Korkina, L. G. Antioxid. Redox Signal 2012, 16, 314-28.

(42) Yao, W.; Feng, D.; Bian, W.; Yang, L.; Li, Y.; Yang, Z.; Xiong, Y.; Zheng, J.; Zhai, R.; He, J. Amino Acids 2012, 43, 2027-35.

(43) Ferguson, K. M.; Darling, P. J.; Mohan, M. J.; Macatee, T. L.; Lemmon, M. A. EMBO J 2000, 19, 4632-43.

(44) Alvarado, D.; Klein, D.E.; Lemmon, M.A. Cell 2010, 142, 568-579.

(45) Greenebaum, E.; Nicolaides, M.; Eisinger, M.; Vogel, R. H.; Weinstein, I. B. J. Natl. Cancer Inst. 1983, 70, 435-41.

(46) Gullick, W. J.; Downward, D. J.; Marsden, J. J.; Waterfield, M. D. Anal. Biochem. 1984, 141, 253-61.

(47) Klein, P.; Mattoon, D.; Lemmon, M. A.; Schlessinger, J. Proc. Natl. Acad. Sci. U S A 2004, 101, 929-34.

(48) Stamos, J.; Sliwkowski, M. X.; Eigenbrot, C. J. Biol. Chem. 2002, 277, 46265-72.

(49) Antczak, C.; Mahida, J.P.; Bhinder, B., Calder, P.A.; Djaballah, H. J. Biomol. Screen. 2012, 17, 885-899.

(50) Kumar, A.; Kumar Singh, U.; Chaudhary, A. Future Med. Chem. 2013, 5, 809-29.

(51) Kunwar, A.; Barik, A.; Mishra, B.; Rathinasamy, K.; Pandey, R.; Priyadarsini, K. I. Biochim. Biophys. Acta 2008, 1780, 673-9.

(52) Harris, F. M.; Best, K. B.; Bell, J. D. Biochim. Biophys. Acta 2002, 1565, 123-8.

(53) Marczak, A. Bioelectrochemistry 2009, 74, 236-9.

(54) Shinitzky, M.; Barenholz, Y. Biochim. Biophys. Acta 1978, 515, 367-94.

(55) Barry, J.; Fritz, M.; Brender, J. R.; Smith, P. E.; Lee, D. K.; Ramamoorthy, A. J. Am. Chem. Soc. 2009, 131, 4490-8.

(56) Eid, M.; Rippa, S.; Castano, S.; Desbat, B.; Chopineau, J.; Rossi, C.; Beven, L. J. Biophys. 2010, 179641.

(57) Kalb, E.; Frey, S.; Tamm, L. K. Biochim. Biophys. Acta 1992, 1103, 307-16.

(58) Boggara, M.; Athmakuri, K.; Srivastava, S.; Cole, R.; Kane, R. S. Biochim. Biophys. Acta 2013, 1828, 419-26.

(59) Otero, C.; Linke, M.; Sanchez, P.; Gonzalez, A.; Schaap, I. A. PLoS One 2013, 8, e83086.

(60) Destainville, N.; Dumas, F.; Salome, L. J. Chem. Biol. 2008, 1, 37-48. 
(61) Chen, A.; Xu, J. Am. J. Physiol. Gastrointest. Liver Physiol. 2005, 288, G447-56.

(62) Sun, X. D.; Liu, X. E.; Huang, D. S. Mol. Med. Rep. 2012, 6, 1267-70.

(63) Reddy, S.; Rishi, A.K.; Xu, H.; Levi, E.; Sarkar, F.H.; Majumdar, A.P.N. Nutr. Cancer 2006, 55, 185-194.

(64) Anand, P.; Kunnumakkara, A. B.; Newman, R. A.; Aggarwal, B. B. Mol. Pharm. 2007, 4, 807-816.

(65) Cheng, A. L.; Hsu, C. H.; Lin, J. K.; Hsu, M. M.; Ho, Y. F.; Shen, T. S.; Ko, J. Y.; Lin, J. T.; Lin, B. R.; Ming-Shiang, W.; Yu, H. S.; Jee, S. H.; Chen, G. S.; Chen, T. M.; Chen, C. A.; Lai, M. K.; Pu, Y. S.; Pan, M. H.; Wang, Y. J.; Tsai, C. C.; Hsieh, C. Y. Anticancer Res. 2001, 21, 2895-900.

(66) Varkondi, E.; Schafer, E.; Bokonyi, G.; Gyokeres, T.; Orfi, L.; Petak, I.; Pap, A.; Szokoloczi, O.; Keri, G.; Schwab, R. J. Recept. Signal Transduct. Res. 2005, 25, 45-56.

(67) Gotink, K. J.; Verheul, H. M. Angiogenesis 2010, 13, 1-14.

14 (68) Khajavi, M.; Shiga, K.; Wiszniewski, W.; He F.; Shaw, C. A.; Yan, J.; Wensel, T. G.;

Snipes, G. J.; Lupski, J. R. Am. J. Hum. Genet. 2007, 81, 438-453.

(69) Minear, S.; O'Donnell, A. F.; Ballew, A.; Giaever, G.; Nislow, C.; Stearns, T.; Cyert, M. S. Eukaryot. Cell 2011, 10, 1574-81. Pharmaceutics 2012, 4, 517-30.

(71) Ingolfsson, H. I.; Koeppe, R. E., 2nd; Andersen, O. S. Biochemistry 2007, 46, 10384-91.

(72) Tanaka, M.; Sackmann, E. Nature 2005, 437, 656-63.

(73) Atsumi, T.; Fujisawa, S.; Tonosaki, K. Oral Dis. 2005, 11, 236-42.

(74) Chen, G.; Chen, Y.; Yang, N.; Zhu, X.; Sun, L.; Li, G. Sci. China Life Sci. 2012, 55, 527532.

(75) Sun, Y.; Lee, C. C.; Hung, W. C.; Chen, F. Y.; Lee, M. T.; Huang, H. W. Biophys. J. 2008, 95, 2318-24.

(76) Pike, L. J.; Casey, L. Biochemistry 2002, 41, 10315-22.

(77) Ringerike, T.; Blystad, F. D.; Levy, F. O.; Madshus, I. H.; Stang, E. J. Cell Sci. 2002, 115, 1331-40.

(78) Westover, E. J.; Covey, D. F.; Brockman, H. L.; Brown, R. E.; Pike, L. J. J. Biol. Chem. 2003, 278, 51125-33.

(79) Ariotti, N.; Liang, H.; Xu, Y.; Zhang, Y.; Yonekubo, Y.; Inder, K.; Du, G.; Parton, R. G.; Hancock, J. F.; Plowman, S. J. Mol. Cell Biol. 2010, 30, 3795-804.

(80) Orr, G.; Hu, D.; Ozcelik, S.; Opresko, L. K.; Wiley, H. S.; Colson, S. D. Biophys. J. 2005, $89,1362-73$. 
1

2

3

4
TABLE OF CONTENTS GRAPHICS

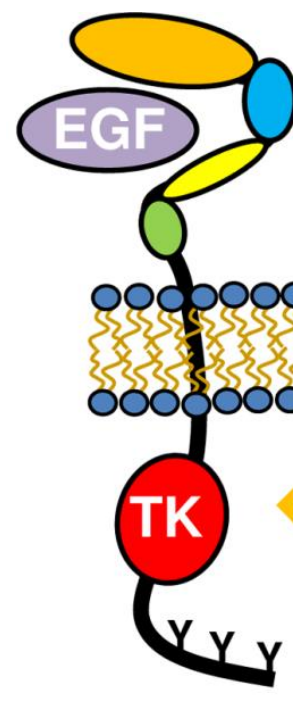

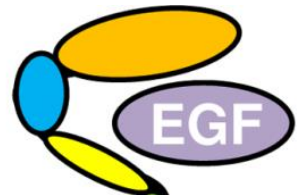

0

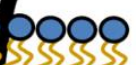

4323

10000

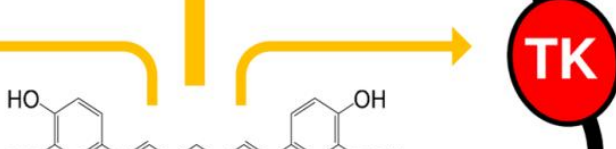

$\mathrm{H}_{3} \mathrm{CO}$

Curcumin 